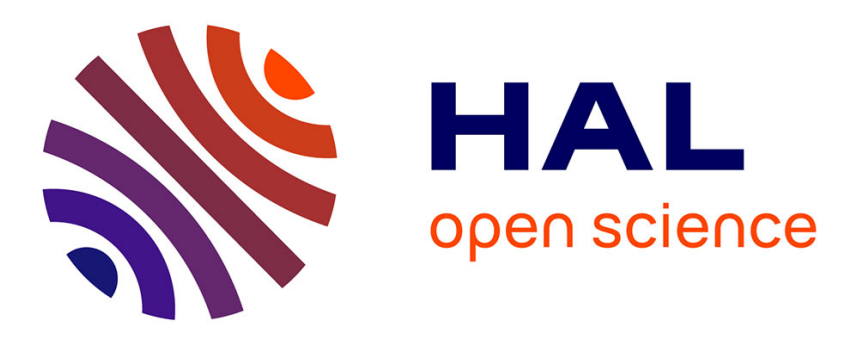

\title{
Comparison of reconstruction approaches for plenoptic imaging systems
}

\author{
Charlotte Herzog, Guillaume Dovillaire, Xavier Granier, Fabrice Harms, \\ Xavier Levecq, Elena Longo, Lois Mignard-Debise, Philippe Zeitoun, \\ Ombeline de La Rochefoucauld
}

\section{To cite this version:}

Charlotte Herzog, Guillaume Dovillaire, Xavier Granier, Fabrice Harms, Xavier Levecq, et al.. Comparison of reconstruction approaches for plenoptic imaging systems. Unconventional Optical Imaging, Apr 2018, Strasbourg, France. pp.106772U:1-106772U:11, 10.1117/12.2306800 . hal-01819610

\section{HAL Id: hal-01819610 https://hal.inria.fr/hal-01819610}

Submitted on 16 Jul 2018

HAL is a multi-disciplinary open access archive for the deposit and dissemination of scientific research documents, whether they are published or not. The documents may come from teaching and research institutions in France or abroad, or from public or private research centers.
L'archive ouverte pluridisciplinaire HAL, est destinée au dépôt et à la diffusion de documents scientifiques de niveau recherche, publiés ou non, émanant des établissements d'enseignement et de recherche français ou étrangers, des laboratoires publics ou privés. 


\title{
Comparison of reconstruction approaches for plenoptic imaging systems
}

\author{
Charlotte Herzog a,b,c , Guillaume Dovillaire ${ }^{\mathrm{a}}$, Xavier Granier ${ }^{\mathrm{b}, \mathrm{c}, \mathrm{d}}$, Fabrice Harms ${ }^{\mathrm{a}}$, Xavier Levecq ${ }^{\mathrm{a}}$, \\ Elena Longo $^{\mathrm{e}}$, Loïs Mignard-Debise ${ }^{\mathrm{b}, \mathrm{c}}$, Philippe Zeitoun ${ }^{\mathrm{e}}$, Ombeline de La Rochefoucauld ${ }^{\mathrm{a}}$ \\ ${ }^{a}$ Imagine Optic, Orsay/Bordeaux, France \\ bLaboratoire Photonique, Numérique et Nanosciences UMR 5298 : Institut d'Optique Graduate \\ School, CNRS, Univ. Bordeaux, France \\ 'Inria Bordeaux, France \\ dLaboratoire Bordelais de Recherche en Informatique UMR5800 : CNRS, Univ. Bordeaux, \\ Bordeaux INP, France \\ 'Laboratoire d'Optique Appliquée UMR7639, ENSTA-CNRS-Ecole Polytechnique- Université \\ Paris-Saclay, Palaiseau, France
}

\begin{abstract}
Plenoptic cameras provide single-shot 3D imaging capabilities, based on the acquisition of the Light-Field, which corresponds to a spatial and directional sampling of all the rays of a scene reaching a detector. Specific algorithms applied on raw Light-Field data allow for the reconstruction of an object at different depths of the scene.

Two different plenoptic imaging geometries have been reported, associated with two reconstruction algorithms: the traditional or unfocused plenoptic camera, also known as plenoptic camera 1.0, and the focused plenoptic camera, also called plenoptic camera 2.0. Both systems use the same optical elements, but placed at different locations: a main lens, a microlens array and a detector. These plenoptic systems have been presented as independent. Here we show the continuity between them, by simply moving the position of an object. We also compare the two reconstruction methods. We theoretically show that the two algorithms are intrinsically based on the same principle and could be applied to any LightField data. However, the resulting images resolution and quality depend on the chosen algorithm.
\end{abstract}

Keywords: Focused Light-Field Camera, Unfocused Light-Field Camera, refocusing, plenoptic imaging, reconstruction algorithm

\section{INTRODUCTION}

Plenoptic camera, also called Light-Field camera, is a type of camera which allows the acquisition of the Light-Field (LF). The LF corresponds to a sampling of both spatial and angular information of all the light rays entering the camera. Compared to traditional photography which acquire a single image focused at a given depth, plenoptic cameras allow processing of the LF after the acquisition, leading to some new applications such as refocusing, changing viewpoint, synthetic aperture, and even 3D reconstruction of the scene. Light-Field imaging has been mainly studied for applications in the visible light such as in photography, in object recognition and in microscopy ${ }^{1,2,3}$. Different kinds of Light-Field cameras have been developed ${ }^{4,5,6}$. In this paper, we focus on plenoptic cameras made of the following three optical elements: a main lens, a microlens array, and a detector. Two main plenoptic designs based on these elements are presented

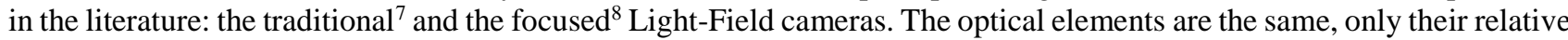
distances differ ${ }^{4}$.

In the literature, these two optical designs and their specific reconstruction algorithms have been presented as independent. The two experimental set-ups have been deeply compared in the literature $9,10,11,6$. But to the best of our knowledge, only Georgiev worked on the link between the two reconstruction approaches, with information spread in several publications $^{8,12,13}$. 
Here, we theoretically demonstrate the continuity between the two experimental designs, by simply moving the position of an object. We compare the reconstruction approaches and show similarities and differences between them. Better understanding of the two plenoptic systems allows to choose the best optical design and the reconstruction algorithm adapted to any sample, in order to fully exploit the acquired data.

\section{OPTICAL DESIGN OF A PLENOPTIC SYSTEM}

The plenoptic camera we consider is composed of a main lens (ML), a microlens array (MLA) and a 2D detector, such as a CCD or CMOS camera, as illustrated Fig. 1. Depending on the distance $(b)$ between MLA and the detector, the configuration is called the unfocused Light-Field camera (ULFC) ${ }^{7}$ or the focused Light-Field camera (FLFC) ${ }^{8}$.

When $b$ equals the focal length $f_{2}$ of the MLA, the design corresponds to an ULFC: the MLA is located on the image plane $Z_{l}$ of an object placed on $Z_{0}$. The distance $Z_{l}$ is given by the thin lens equation:

$$
\frac{1}{Z 0}+\frac{1}{Z 1}=\frac{1}{f 1}
$$

where $f_{l}$ is the focal length of the ML.

When $b$ is not equal to $f_{2}$, a FLFC is obtained: the MLA acts as a relay, focusing on the image plane of the ML. This is where the word Focused Light-Field Camera comes from: the object is first focused on the plane $Z_{l}$ at a distance $a$ from the MLA such that:

$$
\frac{1}{a}+\frac{1}{b}=\frac{1}{f 2}
$$

If $b$ is larger than $f_{2}$, the distance $a$ is considered positive and the intermediate image is located in front of the MLA (ML side), forming a real object for the MLA. If $b$ is smaller than $f_{2}$, the distance $a$ is said negative and the intermediate image is located after the MLA (CCD side), forming a virtual object for the MLA.

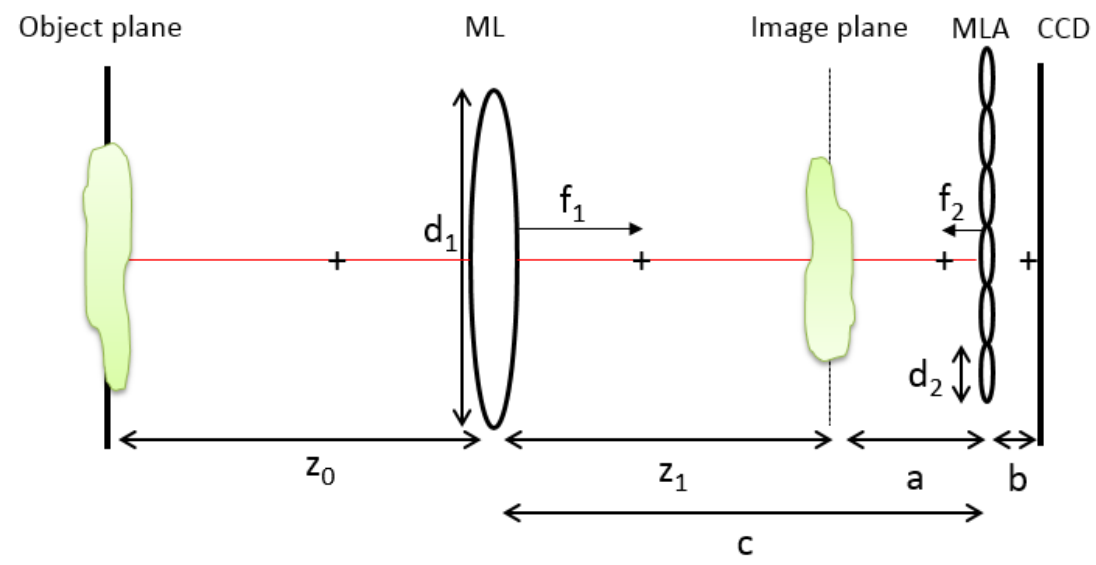

Figure 1: Design of a plenoptic camera, composed of a main lens (ML), of diameter $d_{l}$ and focal length $f_{l}$, of an array of microlenses (MLA) of diameter $d_{2}$ and focal length $f_{2}$ and a detector. The distance $c$ corresponds to the physical distance between the ML and the MLA. An object located on plane $Z_{0}$ is imaged on plane $Z_{l}$ after the ML, at a distance $a$ from the MLA.

Even if the FLFC and ULFC are usually presented as two different systems, we demonstrate here that there is a continuity between the two approaches. By keeping all the distances fixed and simply increasing $Z_{0}$, we can go through three different configurations: FLFC negative $a$ (Fig. 2a), then ULFC (Fig. 2b), and eventually FLFC positive $a$ (Fig. 2c). Theoretically, for each position of the object $Z_{0}$, the distance $b$ should be adjusted accordingly to get a focused raw image. Due to the depth of focus of the microlenses, our plenoptic camera can acquire raw images of the three configurations, without changing the value of $b$. The experimental setup is described in Fig. 2. The corresponding raw images are presented in Figs. 2d-e-f. In the ULFC, the microimages (Fig. 2e) are nearly uniform whereas in the FLFC configuration, the microimages (Fig. 2d, Fig. 1f) correspond to small parts of the scene ${ }^{14,15}$. 
a)

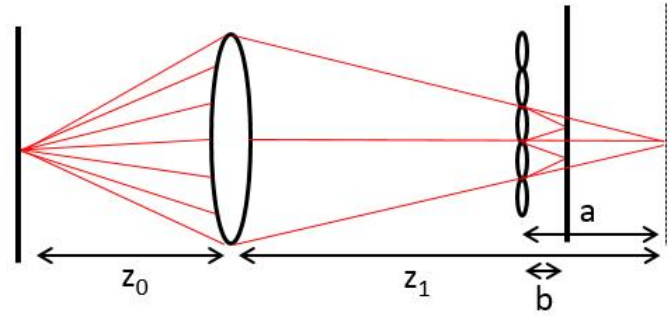

b)

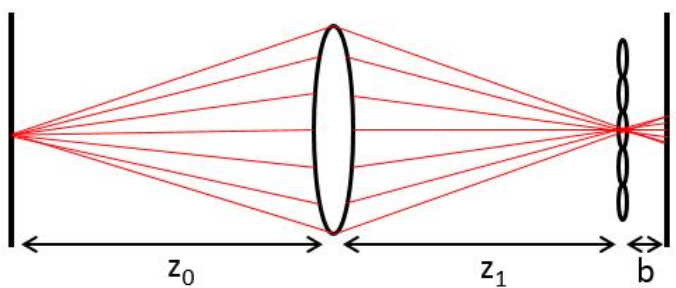

c)

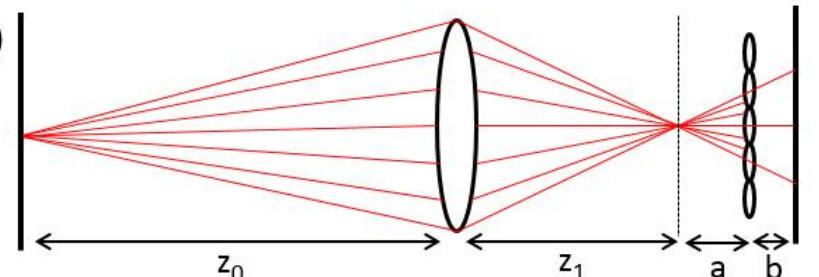

d)

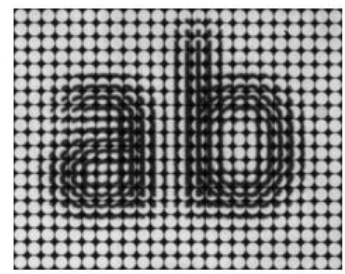

e)
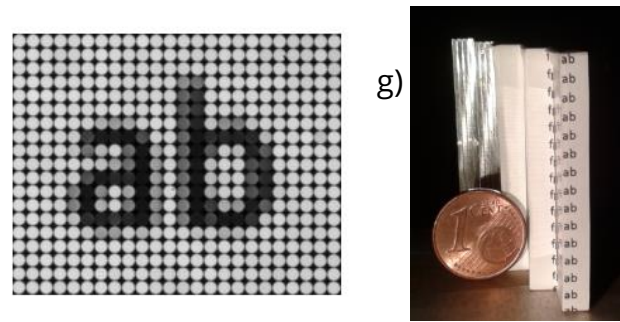

f)

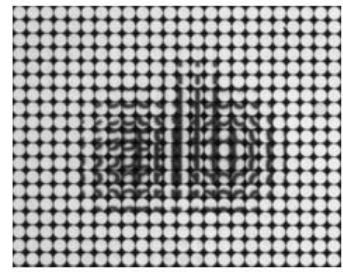

\section{0 increases}

Figure 2: Schematic descriptions of the three different plenoptic configurations and the corresponding raw images.

a) The FLFC configuration with $a<0$, b) the ULFC configuration, c) the FLFC configuration with $a>0$ and d), e), f) the corresponding raw images. g) Printed letters are the imaged object. The ML has a diameter $d_{l}=25 \mathrm{~mm}$ and focal length $f_{l}=$ $200 \mathrm{~mm}$; the MLA is composed of $19 * 24$ microlenses of diameter $d_{2}=300 \mu \mathrm{m}$ and focal length $f_{2}=18,6 \mathrm{~mm}$. The camera is a Stingray F-145 from Allied Vision, with pixel size $6,45 \mu \mathrm{m}$. The distance between ML and MLA is $400 \mathrm{~mm}$ and $b=f_{2}$.

As we have shown the link between the experimental designs, we can expect the two associated reconstruction approaches to be related. This will be discussed in the following section.

\section{COMPARISON OF THE UNFOCUSED AND FOCUSED PLENOPTIC RECONSTRUCTION ALGORITHMS}

Currently each configuration, either FLFC or ULFC, is linked to a specific reconstruction algorithm. We will refer to such algorithms as the Unfocused Light-Field Algorithm (ULFA) and the Focused Light-Field Algorithm (FLFA). It has been empirically shown that it is possible to apply the algorithm from one configuration on data obtained using the other configuration ${ }^{12,13}$. As illustrated in these publications, image quality and resolution depend on the algorithm used.

In this section, we compare the two reconstruction approaches and theoretically show that the two algorithms are intrinsically based on the same principle and could be applied to any Light-Field data. We will clarify the previously observed dependency of the reconstructed image quality on both the algorithm and the optical configuration.

The detector of any plenoptic system captures the Light-Field. It is a parametrization of all the rays passing through both the ML and the MLA. It corresponds to a 4D matrix, LF $(s, t, u, v)$, containing spatial and angular information. For simplicity we will consider the 2D case only, in which LF is a 2D matrix containing only one spatial ( $s$ or $t$-axis) and one angular coordinate ( $u$ or $v$-axis), as illustrated Fig. 3.

The phase-space diagram represents the way a plenoptic camera samples the LF. It depends on the optical configuration (ULFC and FLFC) and can be used to compare the expected performance of the LF acquired with a plenoptic camera ${ }^{9}$. 
The pixel-microlens diagram is a direct representation of the raw data on the CCD, independent from the configuration in which the LF was acquired. To compare the two reconstruction methods (ULFA and FLFA) when applied on the same LF, it is more relevant to study the acquired LF in this pixel-microlens diagram.

\subsection{The Unfocused Light-Field Algorithm}

Let us consider a ray of light crossing the ML at coordinate $(u)$ and reaching the microlenses at coordinate $(s)$, as illustrated in Fig. 3. In a ULFC, information regarding the direction of the ray is given by the coordinate $(u)$, i.e. the position on the ML, while the spatial information is given by the index of the microlens the ray went through (the coordinate $(s)$ ). The MLA plane can also be described in terms of microlenses ( $l$-axis). The step $\Delta s$ between each spatial position is given by the size of a microlens $\left(\Delta s=d_{2}\right)$. Each direction (i.e. each $u$ ) corresponds to only one pixel under the microlens: all the rays coming from this direction will reach the same position of pixel under the microlens. Therefore, the $u$-axis can be described in terms of pixel under a microlens ( $p$-axis) and the step $\Delta u$ can be related to the pixel size $\Delta p$ using similar triangles. There is an inversion between the $u$-axis and the $p$-axis, due to the rays passing through the MLA (Fig. 3). We introduce the parameter $r$ :

$$
r=\frac{\Delta s}{\Delta u}=\frac{d 2}{\Delta p} \frac{b}{Z 1}
$$

We want to reconstruct an image at the refocusing plane $Z{ }^{\prime}{ }_{l}$ corresponding to an object plane $Z{ }^{\prime}$. The refocusing parameter $\alpha$ can be expressed as:

$$
\alpha=\frac{Z \prime 1}{Z 1}=1-\frac{a}{Z 1}
$$

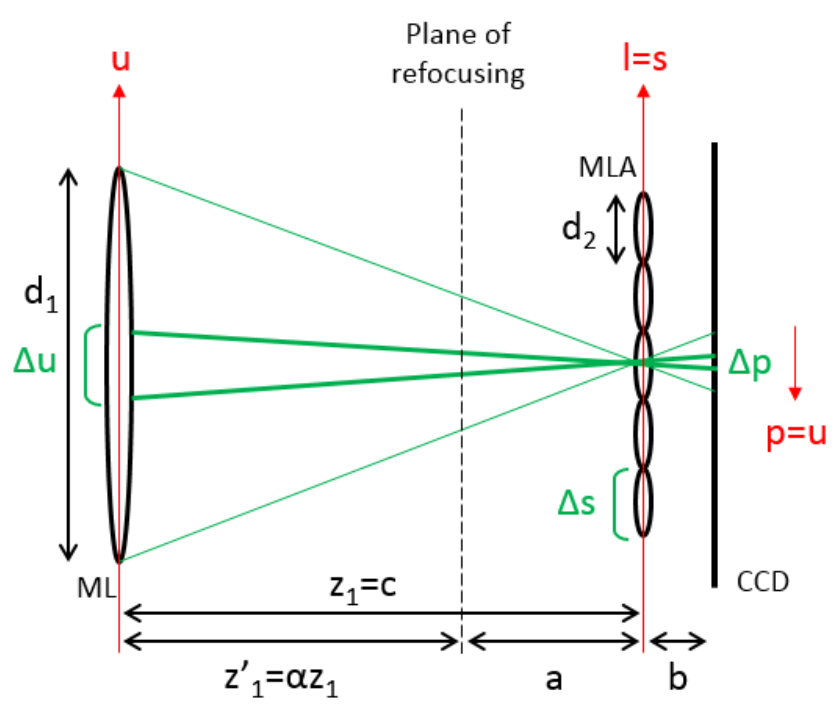

Figure 3: Schematic 2D representation of a plenoptic system. The dashed line represents the position of the image plane $Z_{1}^{\prime}$ to be refocused, with $\alpha$ the refocusing parameter. The plane of the ML is defined by the $u$-axis, the directional axis, while the plane of the MLA corresponds to the $s$-axis, the spatial axis. $\Delta p$ is the pixel size on the detector.

The raw image captured by a ULFC is illustrated in Fig. 4a. From a 1D profile of this raw image, we can determine the 2D LF, as a function of $u$ and $s$ to be implemented in the phase-space diagram (Fig. $4 \mathrm{~b}$ ) or as a function of pixels ( $p$ ) and microlenses $(l)$, as illustrated in the pixel-microlens diagram (Fig. 4c).

In a ULFC, the spatial information is coded by the microlens and the angular information is coded by the pixel under this microlens. In the pixel-microlens diagram, each column represents the pixels $(p)$ under one microlens $(l)$ on the detector. In the phase-space diagram, one position $s$ corresponds to one microlens. One green rectangle corresponds to one pixel underneath the microlens. The dashed green rectangle corresponds to one microlens. The same color coded as in Fig. $4 \mathrm{a}$ was used. Thus, the phase-space diagram and the pixel-microlens diagram are exactly the same (Figs. 4b-c). 


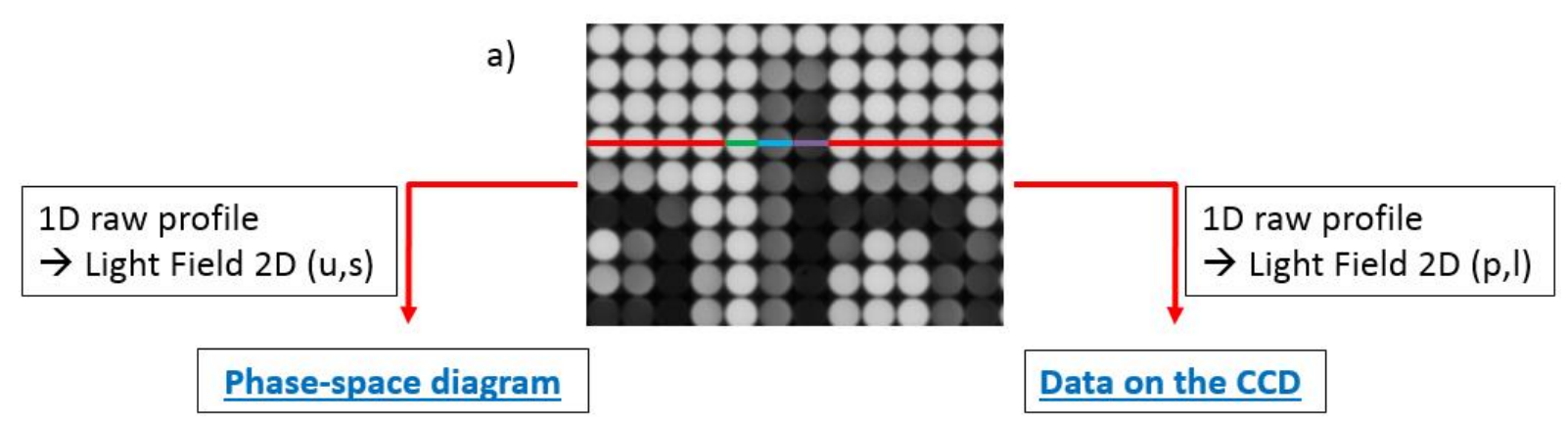

b)

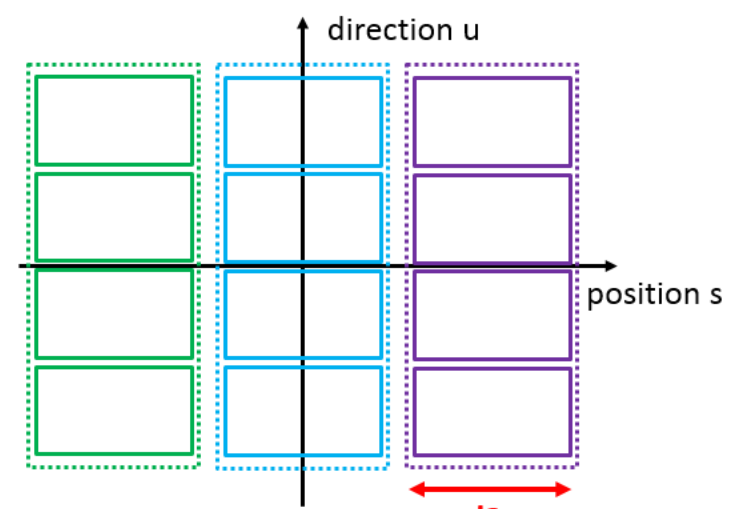

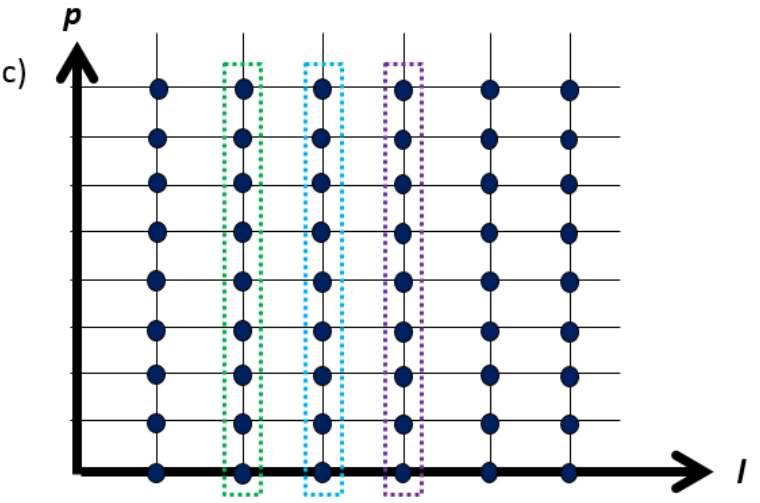

Figure 4: Description of the unfocused configuration. a) Part of a raw image on the CCD; the CCD captures the 4D LF(s,t,u,v); b) phase-space diagram representation of the $2 \mathrm{D} \mathrm{LF}(s, u)$; c) pixel-microlens diagram of the same LF data, $2 \mathrm{D} \operatorname{LF}(l, p)$.

The principle of reconstruction of the ULFA is an integration over the angular information for a fixed spatial coordinate ${ }^{7,4}$. We re-express Eq (2.3) from $\mathrm{Ng}$ 's thesis ${ }^{7}$ as a function of the coordinates of the pixel-microlens diagram, replacing spatial coordinate $(s)$ with microlens $(l)$ and angular coordinate $(u)$ with pixel $(p)$ :

$$
\operatorname{im}_{\alpha}\left(1_{0}\right)=\int L F\left(1_{0}+\mathrm{p} \frac{\alpha\left(1-\frac{1}{\alpha}\right)}{\mathrm{r}}, \mathrm{p}\right) \mathrm{dp}
$$

Compared to the original equation from $\mathrm{Ng}^{7}$, we discard the normalization term before the integration and we introduce the parameter $r$, (Eq. (3)). This parameter is used to compensate the difference in sampling frequency between spatial and angular coordinates. We also multiply the spatial coordinate of the LF by $\alpha: \frac{l_{0}}{\alpha}+p \frac{\left(1-\frac{l}{\alpha}\right)}{r}$ becomes $l_{0}+p \frac{\alpha\left(1-\frac{l}{\alpha}\right)}{r}$. This allows to avoid the zooming/dezooming effect in the reconstructed image when performing refocusing.

Equation (5) means that, to get to the final image ( $\left.i m_{\alpha}\right)$ of an object positioned on a plane $Z^{\prime}{ }^{\prime}$, the LF data have to be shifted and integrated. The parameter $\alpha$ is the refocusing parameter. The case $\alpha=1$ corresponds to a reconstruction at the exact plane where the camera was focused, i.e. the reconstruction of the object placed at a distance $Z_{0}$ in front of the ML. $\alpha \neq 1$, corresponds to refocusing at a different plane (closer to the ML if $\alpha>1$ or farther from the ML if $\alpha<1$ ).

This algorithm can be represented in the pixel-microlens diagram as shown in Fig. 5. It consists in a rotation of the vertical lines (i.e. the microlenses) controlled by the parameter $\alpha$, followed by an integration of the data along these tilted lines (dashed blue arrow in Fig. 5). Two characteristics can be noticed from this diagram. First, the lateral resolution of the reconstructed image, which can be read as the spacing between the lines of integration, is constant and independent from $\alpha$. It corresponds to the spacing of the microlenses, i.e. the diameter of the microlenses, $d_{2}$. Second, as the lines of integration are rotated compared to the initial data, we can see that some points are not part of the initial grid. They can be considered as missing (points with yellow around in Fig. 5) and have to be interpolated to perform an accurate integration. This interpolation across microlenses is a source of errors in the final result. 


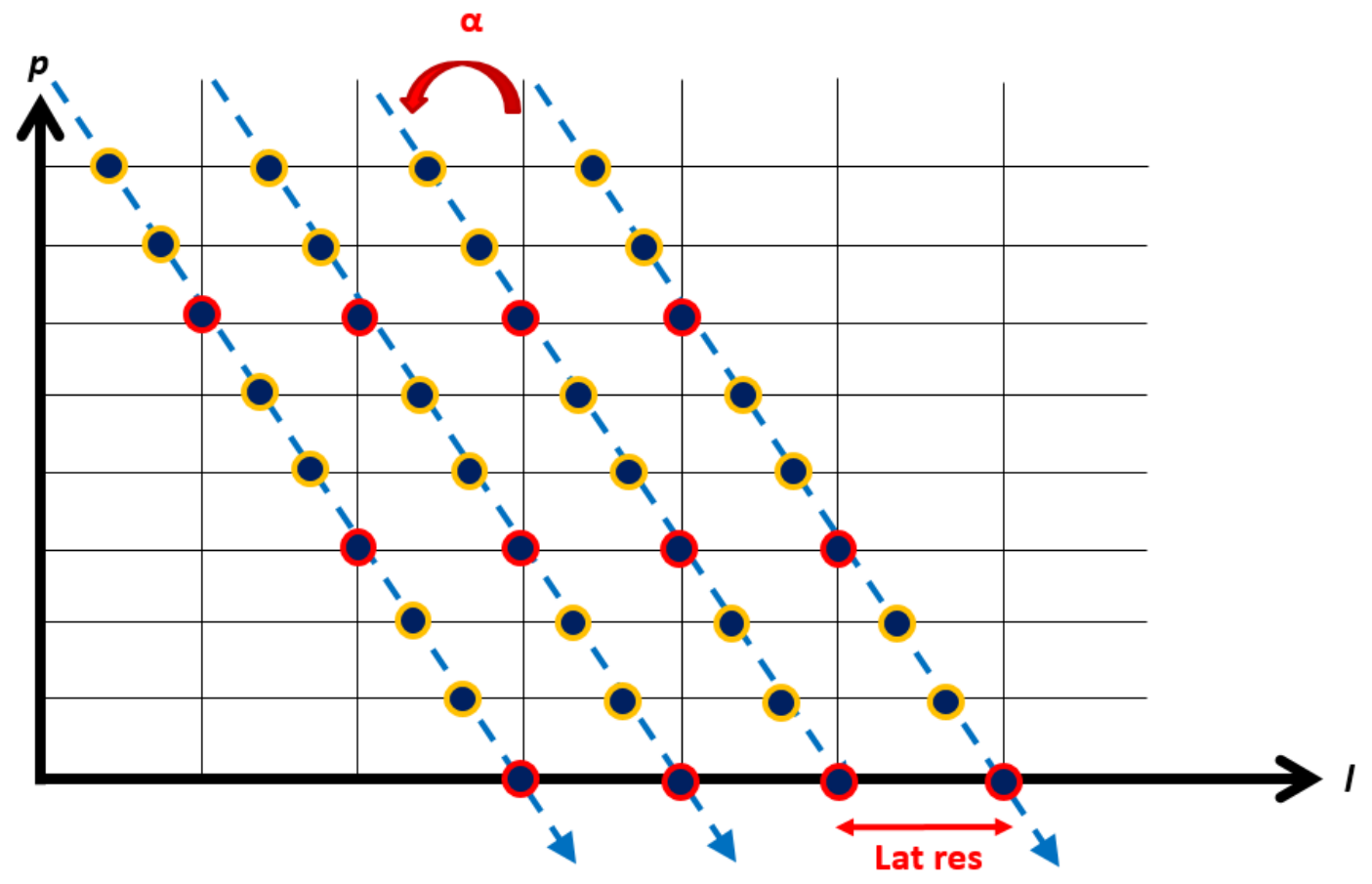

Figure 5: Unfocused plenoptic reconstruction principle in the pixel-microlens diagram. Points with a red circle correspond to pixels acquired by the detector while points circled with yellow represent missing data that has to be created by interpolation. To get the reconstructed image, the integration along the line of equation $l(p)=l_{0}+p \frac{\alpha\left(1-\frac{l}{a}\right)}{r}$ has to be performed, as illustrated by the arrow. We represent here the reconstruction with $\alpha>0$, which corresponds to a negative slope. The lateral resolution of the reconstructed image equals the microlens size.

\subsection{The Focused Light-Field Algorithm}

Part of a raw image captured by a FLFC is illustrated in Fig. 6a. From a 1D raw profile of the acquired data, the 2D LF can be represented as a function of direction and space (phase-space diagram of Fig. 6b) or as a function of pixels and microlenses (as in Fig. 6c). When acquired with a FLFC, the LF has not the same pattern in the phase-space and pixelmicrolens diagram. In the phase-space diagram (Fig. 6b), the microlenses are rotated as shown by the dashed lines ${ }^{9}$, whereas they are still represented as vertical blocks in the pixel-microlens diagram.

As in the previous section, we want to reconstruct a refocused image at a chosen object plane $Z^{\prime}$, which image plane is located on $Z^{\prime}{ }_{l}$ (Fig. 7). If $Z^{\prime}{ }_{l}=Z_{l}$, the refocusing plane corresponds to the exact FLFC image plane of acquisition.

To reconstruct an image, Georgiev presented a basic rendering algorithm ${ }^{16}$. It consists in a patch-tilling method, where a patch of size $P^{*} P$ is extracted from each microimage. The patches are positioned next to each other to form the reconstructed image. $P$ is the parameter of refocusing and is directly related to the depth of the object to reconstruct (Fig. 7). The patch size $P$ is given by the number of pixels necessary to describe a microlens magnified by the MLA. We can express $P$ using similar triangles:

$$
P(a)=\frac{d 2 b}{\Delta p a}
$$




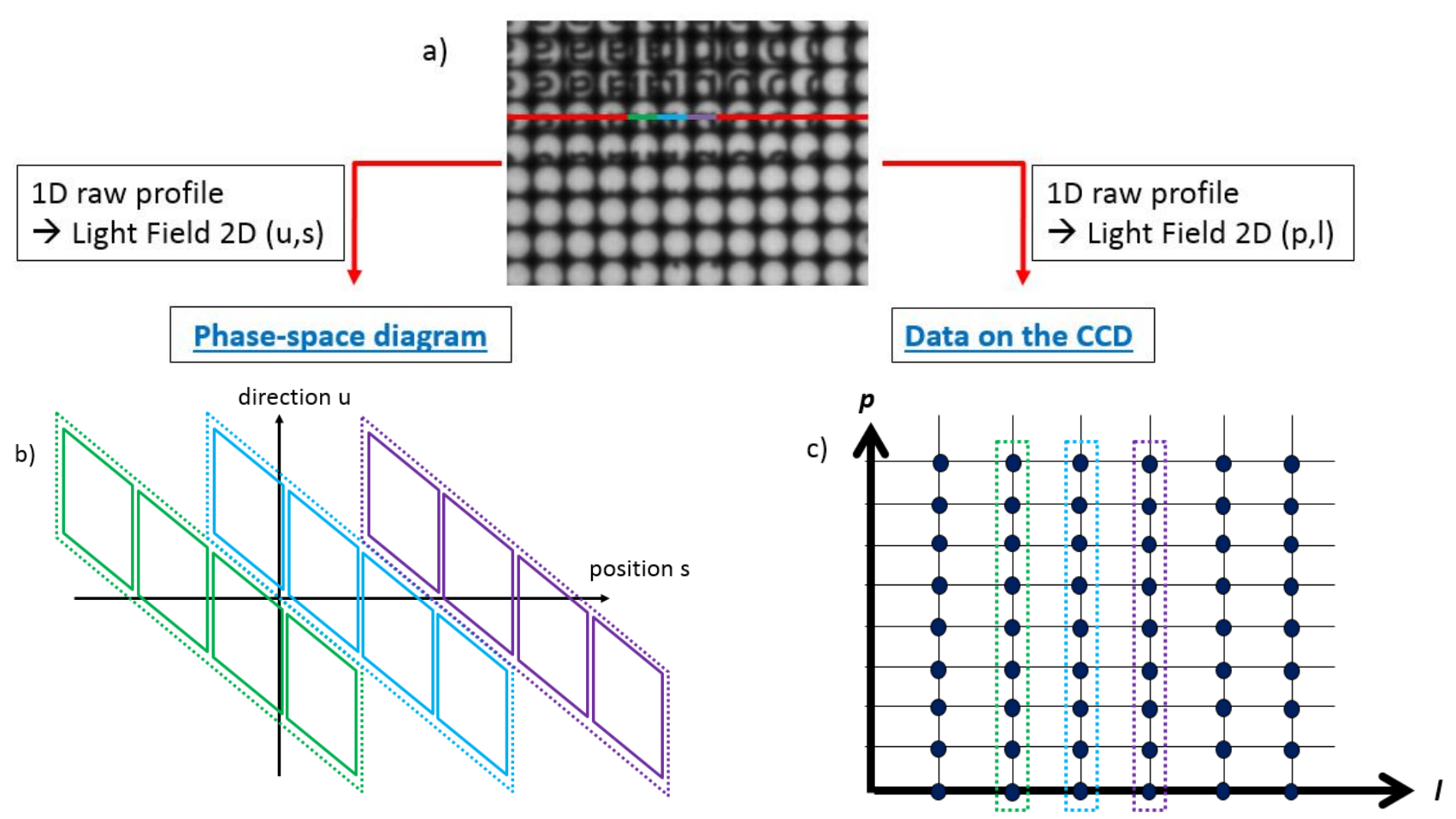

Figure 6: Schematic representation of the Focused Light-Field Camera with a) the raw image, b) the phase-space diagram and c) pixel-microlens representation.

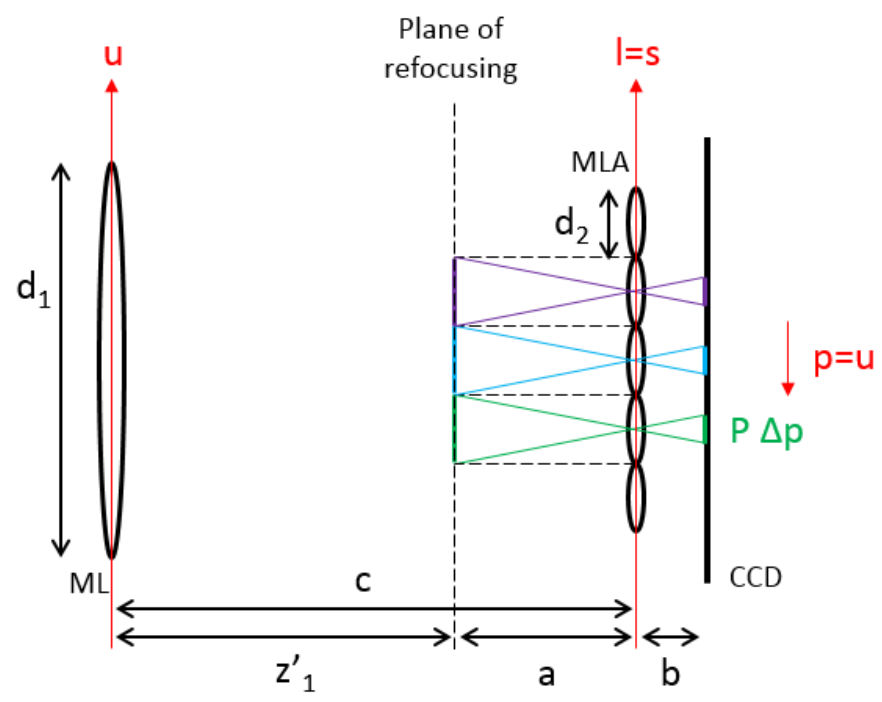

Figure 7: Illustration of the patch size, $P$, the refocusing parameter of the FLFA.

This rendering algorithm uses only the central patches of $P^{*} P$ pixels (in bold in Fig. 8a) from each microimage, ignoring part of the acquired data. Another method, called "rendering with blending" was proposed by Georgiev ${ }^{8}$ and uses an integration. With this method, one blue patch (in Fig. 8a) is associated with other blue patches (taken in the neighbor microimages by shifting the central patches by an integer number of patches, i.e. a multiple of $P$ pixels) and then the integration is performed. All the points used are part of the grid, therefore they were all acquired by the detector. 
When the rendering with blending is performed, each set of associated pixels form a line of integration in the pixelmicrolens diagram (as shown with the blue arrow line in Fig. 8b). The equation of the line of integration can be expressed in terms of pixels $(p)$ and microlens $(l)$ as:

$$
p(l)=p 0-l P
$$

which is equivalent to

$$
l(p)=\frac{p 0-p}{P}
$$

Therefore, the final image associated with the integration method of the FLFC can be defined by the following equation:

$$
\operatorname{im}_{P}(p 0)=\int L F\left(\frac{p 0}{P}-\frac{p}{P}, p\right) d p
$$

a)
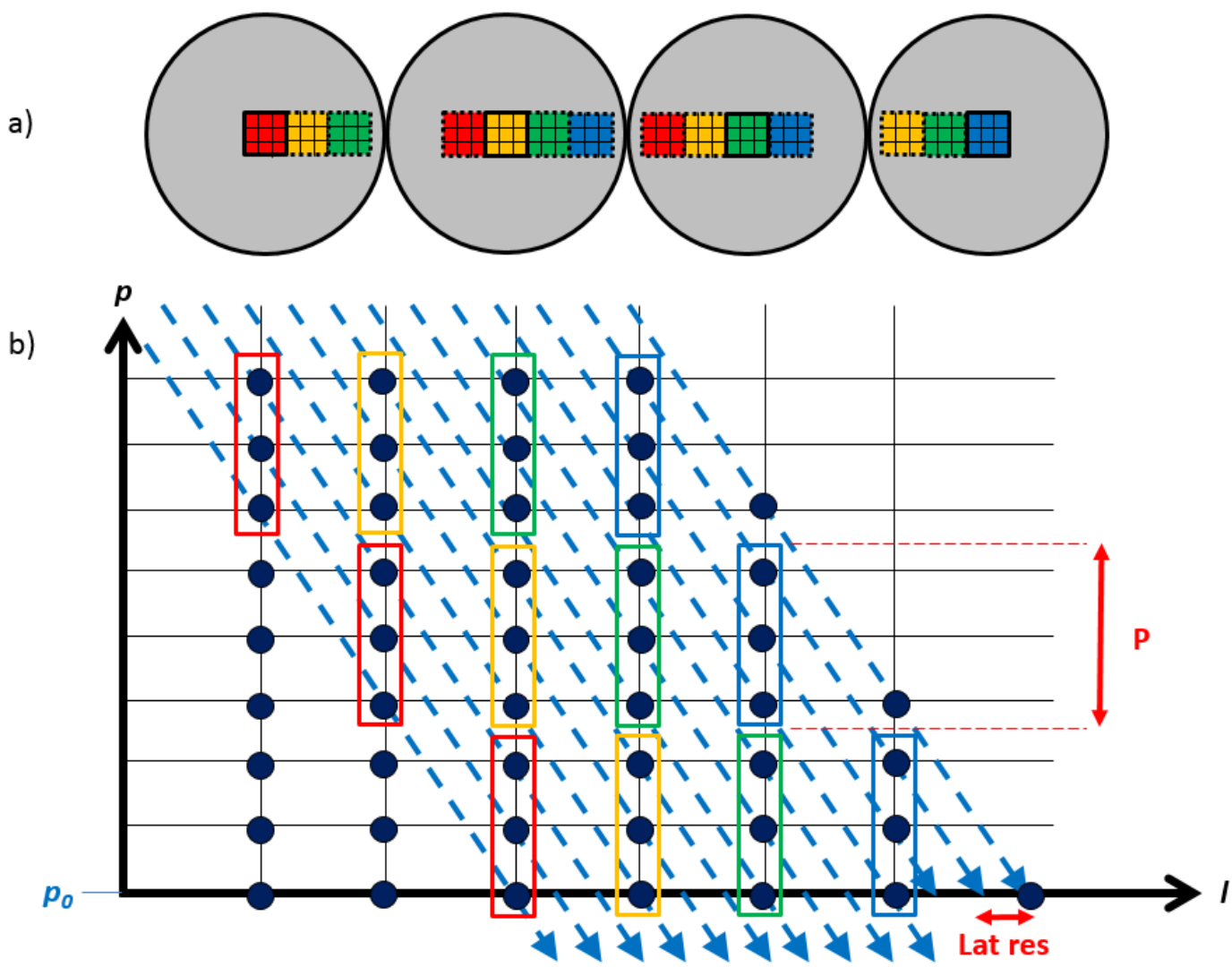

Figure 8: a) Illustration of patches composed of $3 * 3$ pixels and their correspondence with neighboring microimages. b) Pixelmicrolens representation of the integration method for the FLFC following a line of equation $l(p)=(p 0-p) / P$. We consider a reconstruction in the case $a>0$, leading to $P>0$ and a negative slope.

\subsection{Comparison of the two algorithms}

Writing the FLFA in the same diagram as the ULFA allows to compare the two algorithms when applied on the same raw data. In this diagram, it is easier to notice that not only the ULFA but both algorithms correspond to integration along straight lines in the pixel-microlens diagram ${ }^{8,11}$.

To go even further in the comparison, let us consider the case where we want to refocus on an object located on plane $Z^{\prime}{ }^{\prime}$, with its image on plane $Z_{1}^{\prime}$, with both algorithm independently (Figs. 3 and 7). The slope of integration of the FLFA (Eq. 
9) is given by $\frac{-1}{P}$, while the slope for the ULFA (Eq. 5) equals $\frac{\alpha\left(1-\frac{1}{\alpha}\right)}{r}$. From the expressions of $r, \alpha$ and $P($ Eqs. $3 ; 4$ and 6), we notice that the two slopes are equals:

$$
\frac{\alpha\left(1-\frac{1}{\alpha}\right)}{\mathrm{r}}=\frac{-1}{\mathrm{P}}
$$

This means that the orientation of the lines is exactly the same for both algorithms when choosing the same plane of refocusing. Therefore, the main similarity of the two algorithms is that they perform integration over lines with exactly the same slope.

Knowing that the principle of reconstruction of the two methods is basically equivalent, we wanted to compare their output by applying them on two raw images, one obtained using an ULFC and the other one using a FLFC. The results are presented in Fig. 9.

For the raw image acquired with the ULFC, there is nearly no difference between the results obtained with the two different reconstruction algorithms (Fig. 9: images on the top). For the image acquired with the FLFC (Fig. 9: images at the bottom), we can notice a difference in resolution and image quality between the images resulting from the two different algorithms. The reconstructed FLFC image by the ULFA is of the same quality than the reconstructed ULFC one, whereas the reconstructed FLFC image using the FLFA shows better resolution and image quality.

\begin{tabular}{|l|l|l|}
\hline & $\begin{array}{l}\text { Reconstruction with the Unfocused Light- } \\
\text { Field algorithm }\end{array}$ & $\begin{array}{l}\text { Reconstruction with the Focused Light- } \\
\text { Field algorithm }\end{array}$ \\
\hline $\begin{array}{l}\text { Image acquired } \\
\text { with an ULFC } \\
\text { (M=2.2) }\end{array}$ & & \\
\hline $\begin{array}{l}\text { Image acquired } \\
\text { with a FLFC } \\
\text { (M=1.67) }\end{array}$
\end{tabular}

Figure 9: Comparison of images taken with an ULFC and a FLFC and retrieved using both reconstruction methods.

The difference in resolution can be explained mathematically by considering both pixel-microlens diagrams. With the ULFA, the lateral resolution is given by the size of a microlens, $d_{2}$, while for the FLFA, the lateral resolution equals $d_{2} / P$. FLFA generates more lines of integration hence more pixels in the reconstructed image. We may thus establish the relation between resolutions with FLFA and ULFA (Eq. 11). As $P$ is usually larger than 1, this formula explains the finest resolution of the FLFA:

$$
\text { Lat res }(F L F A)=\frac{\text { Lat res }(U L F A)}{P}
$$


We believe that the difference in image quality observed with the FLFC raw image can be explained by the interpolation step in the ULFA. In the FLFA, only points acquired by the detector are used for the integration over the lines to get the final image while for the ULFA, points have to be interpolated to get to the final image.

The lack of difference between the two reconstructed images from the ULFC raw data can be explained by the configuration itself. In the ULFC, the LF is acquired such that the microlens size restricts the resolutions. Using the ULFA or the FLFA does not change the resolution of the reconstructed image for data acquired in this configuration.

\section{CONCLUSION}

As a conclusion, this work presented the experimental continuity between the ULFC and the FLFC. It illustrates that the plenoptic camera composed of a ML, a MLA and a detector is a unique optical concept, independent of the relative position of the optics. We have also shown that although the two reconstruction methods associated to the ULFC and the FLFC were presented as independent, their principles are in fact very close. They consist on the integration along a line, with a slope depending on the position of the plane of refocusing. This means that both algorithms can be applied independently on raw images acquired either with an ULFC or a FLFC. However, the resulting images resolution and quality depend on the chosen algorithm. Therefore, the choice of an optimal algorithm allows to fully exploit the acquired data and optimize the $3 \mathrm{D}$ reconstruction in terms of resolution and image quality. These algorithms could be improved. For example, Georgiev $^{13}$ developed an algorithm based on super-resolution techniques, allowing to increase by a factor 9 the spatial resolution compared to the basic rendering approach.

\section{ACKNOWLEDGEMENTS}

This work was funded by the VOXEL project (European Union's Horizon 2020 research and innovation program under grant agreement $\mathrm{N}^{\circ}$ 665207).

\section{REFERENCES}

[1] Levoy, M., Ng, R., Adams, A., Footer, M., Horowitz, M., Levoy, M., Ng, R., Adams, A., Footer, M. and Horowitz, M., "Light field microscopy," ACM Trans. Graph. 25(3), 924, ACM Press, New York, New York, USA (2006).

[2] Bedard, N., Shope, T., Hoberman, A., Haralam, M. A., Shaikh, N., Kovačević, J., Balram, N. and Tošić, I., "Light field otoscope design for 3D in vivo imaging of the middle ear.," Biomed. Opt. Express 8(1), 260-272 (2017).

[3] Mignard-Debise, L. and Ihrke, I., "Light-field Microscopy with a Consumer Light-field Camera," 3D Vis. 2015, 335-343 (2015).

[4] Lam, E. Y., "Computational photography with plenoptic camera and light field capture: tutorial,” J. Opt. Soc. Am. A 32(11), 2021-2032 (2015).

[5] Ihrke, I., Restrepo, J. and Mignard-Debise, L., "Principles of Light Field Imaging: Briefly revisiting 25 years of research,” IEEE Signal Process. Mag. Inst. Electr. Electron. Eng. 33(5), 59-69 (2016).

[6] Cossu, K., Druart, G., Montmerle Bonnefois, A. and Velluet, M.-T., "Caméras plénoptiques pour l'imagerie tridimensionnelle," Tech. l'ingénieur Mes. tridimensionnelles états Surf. TIB409DUO(r1393) (2016).

[7] Ng, R., "Digital light field photography," Stanford Univ. (2006).

[8] Georgiev, T. and Lumsdaine, A., "Focused plenoptic camera and rendering," J. Electron. Imaging 19(2), 21106 (2010).

[9] Lumsdaine, A., Georgiev, T. G. and Chunev, G., "Spatial analysis of discrete plenoptic sampling," SPIE Electron. Imaging 8299, 829909 (2012).

[10] Zhu, S., Lai, A., Eaton, K., Jin, P. and Gao, L., "On the fundamental comparison between unfocused and focused 
light field cameras," Appl. Opt. 57(1), A1-A11 (2018).

[11] Turola, M., "Investigation of plenoptic imaging systems: a wave optics approach" (2016).

[12] Lumsdaine, A. and Georgiev, T., "The Focused Plenoptic Camera," Comput. Photogr. (ICCP), 2009 IEEE Int. Conf., 1-8 (2009).

[13] Georgiev, T. and Lumsdaine, A., "Superresolution with Plenoptic Camera 2.0," Adobe Tech. Rep. (2009).

[14] Georgiev, T. and Lumsdaine, A., "Depth of Field in Plenoptic Cameras," Eurographics (2009).

[15] Zhang, C., Ji, Z. and Wang, Q., "Decoding and calibration method on focused plenoptic camera," Comput. Vis. Media 2(1), 57-69 (2016).

[16] Lumsdaine, A. and Georgiev, T., "Full Resolution Lightfield Rendering," Adobe Tech Rep., 1-12 (2008). 